\title{
Time Domain Early Arrival Waveform Inversion
}

Jie Zhang, GeoTomo, Houston, Texas, USA

Seismic early arrivals recorded on the surface include sufficient velocity structural information in the near-surface area. Unlike later arrivals, they are less contaminated by the elastic effects such as surface waves or converted waves. Thus, the early arrivals can be better delineated as an acoustic wave propagation problem. Time domain early arrival waveform inversion by applying an acoustic finite-difference method to simulate a small window of wavefield, and employing a conjugate gradient method to invert the shallow near-surface velocity structure is then more computationally efficient and practical than the full waveform inversion that intends to invert the entire subsurface velocity model. It could help to resolve complex near-surface velocity structures that cannot be inverted by just traveltimes alone. However, time domain early arrival waveform inversion also faces with a number of serious challenges. They include: 1) numerical simulation accuracy to handle the surface topography effects in the forward finite-difference modeling; 2 ) velocities too low in the nearsurface area, requiring substantial small grids for stable finite-difference simulation; 3) large amplitude variations along the surface due to the near-surface attenuation and various complex structural effects. To address these issues, we developed a variable-grid finite-difference approach for forward modeling, and stable shot-based amplitude balancing algorithm to process both data and synthetics in time domain. Numerical and real data examples demonstrate that the waveform inversion method can handle low velocity zones, hidden layers, and strong lateral velocity variations. 